\title{
Actualización con la clasificación de Londres en la interpretación de la manometría anorrectal de alta resolución
}

\section{Update of high-resolution anorectal manometry interpretation using the London classification}

\author{
Andrés Delgado Villarreal ${ }^{1 *}$, Valeria Atenea Costa Barney², Albis Hani², Ana María Leguizamo², Andrés Ardila², Diana Meza Madrid ${ }^{1}$
}

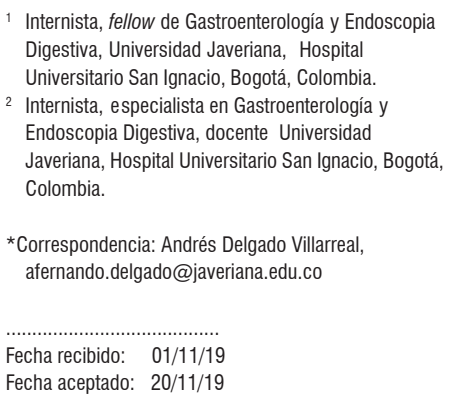

\begin{abstract}
Resumen
La manometría anorrectal de alta resolución es una prueba diagnóstica utilizada para los trastornos motores y sensitivos anorrectales. Consta de una medición del tono basal y de contracción anal; también valora la maniobra de pujo, reflejo rectoanal inhibitorio (RIAR) y parámetros sensitivos rectales. La interpretación convencional de la manometría anorrectal se enfoca en describir aisladamente la región anatómica disfuncional. Sin embargo, con la clasificación de Londres se busca estandarizar el informe de estos resultados, agrupándolos en trastornos mayores, menores y hallazgos no concluyentes, similar a la clasificación de Chicago para trastornos motores esofágicos.
\end{abstract}

\section{Palabras clave}

Manometría anorrectal, trastornos funcionales anorrectales, fisiología anorrectal, clasificación de Londres.

\section{Abstract}

High resolution anorectal manometry is a diagnostic test, used for anorectal motor and sensory disorders. It consists of measurement of basal tone, anal contraction and squeeze, the rectoanal inhibitory reflex (RAIR), and rectal sensory parameters. The conventional interpretation of anorectal manometry focuses on describing the dysfunctional anatomical region in isolation. However, the London classification seeks to standardize the report of these results, grouping them into major, minor and inconclusive findings in a manner similar to the Chicago classification for esophageal motor disorders.

\section{Keywords}

Anorectal manometry, anorectal functional disorders, anorectal physiology, London classification.

\section{INTRODUCCIÓN}

En los trastornos anorrectales, la incontinencia fecal y el estreñimiento son los más frecuentes (1). Pueden originarse de alteraciones estructurales o funcionales y, en el enfoque inicial, si bien son importantes los síntomas y el examen físico, no alcanzan a ser suficientes para determinar la causa, siendo necesario complementar con estudios de imágenes, como la defecografía por resonancia o fluoroscopia, tránsito colónico, endosonografía rectoanal y manometría anorrectal de alta resolución (2-4).
Desde 1999, la Asociación Americana de Gastroenterología (AGA) introduce la utilidad de la manometría anorrectal, pruebas de sensibilidad rectal y de expulsión de balón en la aproximación diagnóstica para la evaluación del esfínter anal y de la coordinación anorrectal (5). Sin embargo, a lo largo de estos años no se ha logrado estandarizar su realización e interpretación, por lo que la validez externa de los estudios se ve afectada, lo que altera los resultados diagnósticos de esta prueba (6-8). Es por esto por lo que el International Anorectal Physiology Working Group (IAPWG) propone una estandarización 
de la manometría anorrectal mediante la clasificación de Londres publicada en 2019 (9).

\section{EPIDEMIOLOGÍA}

Se ha descrito en países desarrollados una prevalencia global de estreñimiento e incontinencia cercana al 20\% (10). Sin embargo, se debe evaluar con cuidado, ya que depende de los criterios diagnósticos utilizados para estas dos patologías, además de la edad del paciente y sitio de realización del estudio, como ambulatorio, hospitalización y hogar geriátrico. Es así como en población mayor de 60 años se ha descrito una prevalencia del $9,9 \%$ de incontinencia fecal y hasta del $20 \%$ de estreñimiento, siendo más frecuente en las personas que viven en hogares geriátricos, con una prevalencia hasta del $50 \%(11,12)$.

\section{INDICACIONES DE LA MANOMETRÍA ANORRECTAL}

Las principales indicaciones de la manometría anorrectal son el estreñimiento y la incontinencia fecal, pero hay otras indicaciones menos comunes, como el dolor anorrectal, megacolon y megarrecto $(1,14,15)$. En la Tabla 1 se describe a mayor detalle las indicaciones de la manometría anorrectal de alta resolución.
Tabla 1. Indicaciones para la realización de manometría anorrectal, prueba de sensibilidad rectal y expulsión de balón

\begin{tabular}{ll}
\hline \multicolumn{1}{c}{ Indicación } & \multicolumn{1}{c}{$\begin{array}{c}\text { Parámetros de las pruebas a } \\
\text { evaluar }\end{array}$} \\
\hline $\begin{array}{l}\text { Estreñimiento } \\
\text { Megarrecto/megacolon }\end{array}$ & $\begin{array}{l}\text { Coordinación anorrectal } \\
\text { (disinergias y prueba anormal de } \\
\text { expulsión de balón) } \\
\text { Hiposensibilidad rectal } \\
\text { Ausencia de RIAR } \\
\text { Incontinencia fecal }\end{array}$ \\
Holor anorrectal funcional del esfínter anal \\
Hipo/hipersensibilidad rectal \\
Hipertensión del esfínter anal \\
Coordinación anorrectal \\
(disinergias y prueba anormal de \\
expulsión de balón) \\
Previo a cirugía anorrectal & $\begin{array}{l}\text { Función del esfínter anal } \\
\text { Coordinación anorrectal }\end{array}$ \\
Antecedente de lesión obstétrica & Función del esfínter anal \\
\hline
\end{tabular}

RIAR: reflejo rectoanal inhibitorio.

\section{CLASIFICACIÓN DE LONDRES EN TRASTORNOS ANORRECTALES}

En agosto de 2019, el LAPWG, conformado por 29 gastroenterólogos, coloproctólogos y fisiólogos de 12 paí-

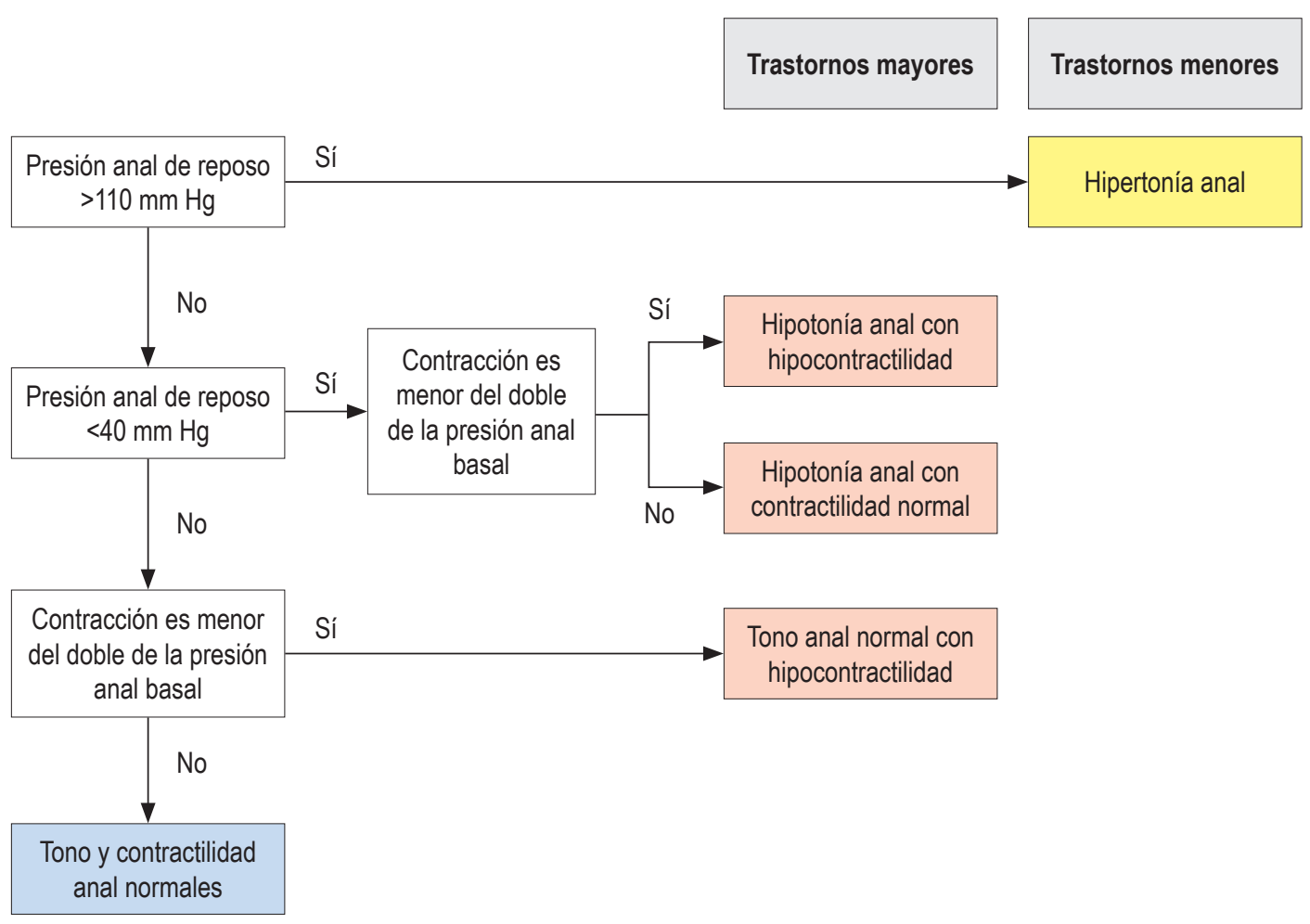

Hallazgos no concluyentes

Figura 1. Trastornos del tono y la contractilidad anal (9). 
ses, presentaron el primer protocolo mediante el cual se busca estandarizar la realización de la manometría anorrectal de alta resolución, las pruebas de sensibilidad rectal y expulsión de balón. Esta se propone como la clasificación de Londres para la interpretación de los resultados (9).

Con el fin de tener una adecuada realización y reproducibilidad de esta prueba diagnóstica, Londres continua recomendando el uso del estándar propuesto por la IAPWG, que consta de una estabilización de 3 minutos del sensor, toma de presión del esfínter anal en reposo por 60 segundos, tres maniobras de contracción de 5 segundos cada una, contracción prolongada de 30 segundos, dos maniobras de tos simple, tres maniobras de pujo de 15 segundos cada una, medición de sensibilidad rectal mediante la distensión con balón a volúmenes progresivos, RIAR y, finalmente, la prueba de expulsión de balón (15).

La clasificación de Londres se centra en cuatro grandes grupos de alteraciones anorrectales que, teniendo en cuenta el protocolo de la IAPWG, proponemos sean revisados en el siguiente orden: tono y contractilidad anal (Figura 1), coordinación anorrectal (Figura 2), sensibilidad rectal (Figura 3) y, por último, el reflejo rectoanal inhibitorio (RIAR) (Figura 4).
No contamos con estudios grandes en nuestra población para establecer los valores de normalidad en cuanto a la presión anal de reposo, presión de contracción y presión rectal; igualmente, tampoco se disponen de valores para la relajación anal y parámetros de sensibilidad rectal, por lo que estos son tomados de estudios internacionales, con población de gran diversidad y los adaptamos para la clasificación de Londres (9, 16-19).

$\mathrm{Al}$ igual que la clasificación de Chicago para trastornos de la motilidad esofágica, en Londres se clasifican los trastornos anorrectales en mayores, menores y de hallazgo no significativo, según su relevancia patológica, respectivamente (20). En la Tabla 2 se realiza una comparación entre la nueva propuesta de interpretación de la manometría anorrectal y la convencional.

\section{CONCLUSIÓN}

La manometría anorrectal es una herramienta diagnóstica útil en los trastornos sensitivo-motores anorrectales. La adecuada realización e interpretación son fundamentales para lograr brindar finalmente al paciente un adecuado tra-

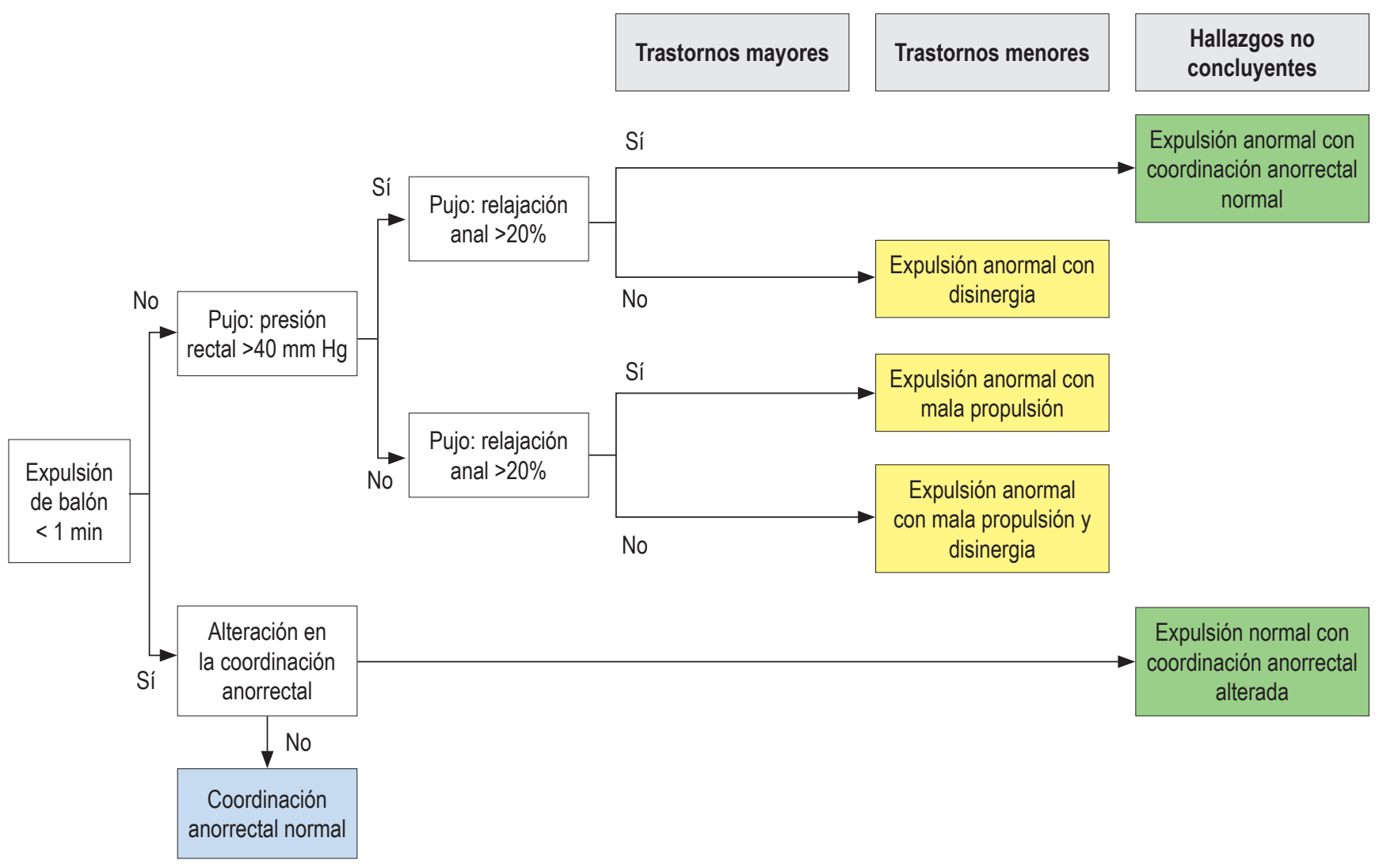

Figura 2. Trastornos de la coordinación anorrectal (9). 


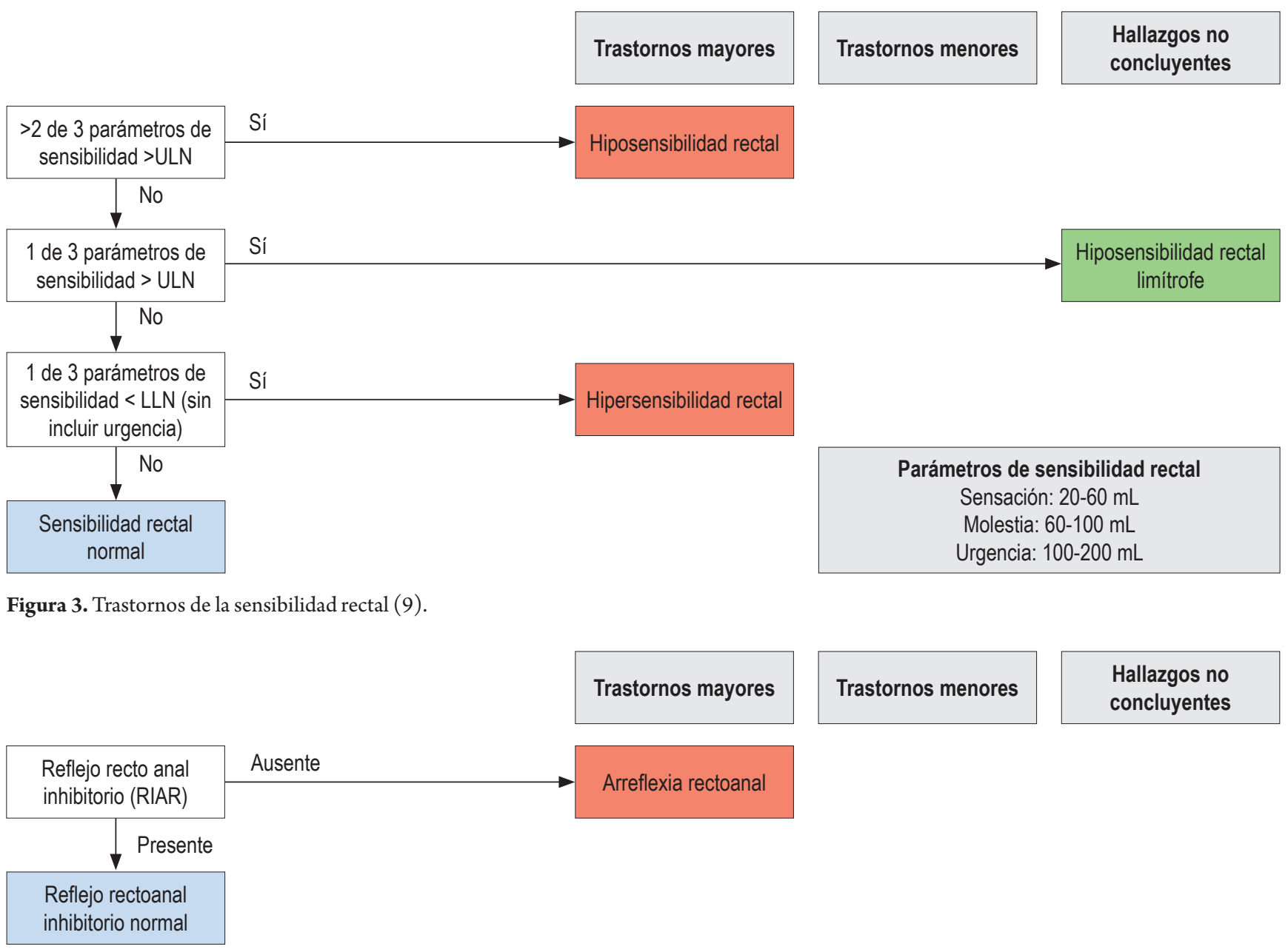

Figura 4. Trastornos del reflejo rectoanal inhibitorio (RIAR) (9).

Tabla 2. Comparación entre la clasificación de Londres y la nomenclatura convencional de la manometría anorrectal de alta resolución

\begin{tabular}{ll}
\hline \multicolumn{1}{c}{ Clasificación de Londres } & \multicolumn{1}{c}{ Nomenclatura convencional } \\
\hline Trastornos mayores & Alteración (hipotonía) del esfínter anal interno. Alteración del esfínter anal externo \\
Hipotensión anal con hipocontractilidad & Alteración (hipotonía) del esfínter anal interno \\
Hipotensión anal con contractilidad normal & Alteración o disfunción del esfínter anal externo \\
Tono anal normal con hipocontractilidad & Trastornos de la sensibilidad tipo hiposensibilidad rectal \\
Hiposensibilidad rectal & Trastornos de la sensibilidad tipo hipersensibilidad rectal \\
Hipersensibilidad rectal & Reflejo rectoanal inhibitorio ausente \\
Arreflexia rectoanal & \\
Trastornos menores & Esfínter anal hipertónico \\
Hipertensión anal & Disinergia defecatoria tipo I o tipo III \\
Expulsión anormal con disinergia & No aplica \\
Expulsión anormal con pobre propulsión & Disinergia defecatoria tipo II o tipo IV \\
Expulsión anormal con pobre propulsión y disinergia & \\
Trastornos no concluyentes & Expulsión anormal de balón \\
Expulsión anormal con coordinación anorrectal normal & Disinergias defecatorias tipo I-II-III-IV \\
Expulsión normal con coordinación anorrectal alterada & No aplica \\
Hiposensibilidad rectal limítrofe &
\end{tabular}


tamiento. La clasificación de Londres es la primera propuesta que busca estandarizar el reporte de resultados de la manometría anorrectal de alta resolución.

\section{REFERENCIAS}

1. Bharucha AE, Rao SS. An update on anorectal disorders for gastroenterologists. Gastroenterology. 2014;146(1):37-45. doi: https://doi.org/10.1053/j.gastro.2013.10.062.

2. Patcharatrakul T, Rao SSC. Update on the pathophysiology and management of anorectal disorders. Gut Liver. 2018;12(4):375-84. doi: https://doi.org/10.5009/gnl17172.

3. Rao SS, Bharucha AE, Chiarioni G, Felt-Bersma R, Knowles C, Malcolm A, et al. Functional anorectal disorders. Gastroenterology. 2016;S0016-5085(16)00175-X. doi: https://doi.org/10.1053/j.gastro.2016.02.009.

4. Nuernberg D, Saftoiu A, Barreiros AP, Burmester E, Ivan ET, Clevert DA, et al. EFSUMB recommendations for gastrointestinal ultrasound part 3: endorectal, endoanal and perineal ultrasound. Ultrasound Int Open. 2019;5(1):E34-51. doi: https://doi.org/10.1055/a-0825-6708.

5. Diamant NE, Kamm MA, Wald A, Whitehead WE. AGA technical review on anorectal testing techniques. Gastroenterology. 1999;116(3):735-60. doi: https://doi. org/10.1016/S0016-5085(99)70195-2.

6. Heinrich H, Fruehauf H, Sauter M, Steingötter A, Fried M, Schwizer W, et al. The effect of standard compared to enhanced instruction and verbal feedback on anorectal manometry measurements. Neurogastroenterol Motil. 2013;25(3):2307,e163. doi: https://doi.org/10.1111/nmo.12038.

7. Basilisco G, Bharucha AE. High-resolution anorectal manometry: an expensive hobby or worth every penny? Neurogastroenterol Motil. 2017;29(8). doi: https://doi. org/10.1111/nmo.13125.

8. Carrington EV, Heinrich $\mathrm{H}$, Knowles $\mathrm{CH}$, Rao SS, Fox M, Scott SM, et al. Methods of anorectal manometry vary widely in clinical practice: results from an international survey. Neurogastroenterol Motil. 2017;29(8):e13016. doi: https://doi.org/10.1111/nmo.13016.

9. Carrington EV, Heinrich $\mathrm{H}$, Knowles $\mathrm{CH}$, Fox $\mathrm{M}$, Rao $\mathrm{S}$, Altomare DF, et al. The International Anorectal Physiology Working Group (IAPWG) recommendations: standardized testing protocol and the London classification for disorders of anorectal function. Neurogastroenterol Motil. 2019:e13679. doi: https://doi.org/10.1111/nmo.13679.

10. Remes-Troche JM, Rao SS. Defecation disorders: neuromuscular aspects and treatment. Curr Gastroenterol Rep. 2006;8:2919. doi: https://doi.org/10.1007/s11894-006-0049-x.

11. Demir N, Yuruyen M, Atay K, Yavuzer H, Hatemi I, Doventas A, et al. Prevalence of fecal incontinence and associated risk factors in elderly outpatients: a cross-sectional study. Aging Clin Exp Res. 2017;29(6):1165-71. doi: https://doi. org/10.1007/s40520-017-0723-x.

12. Vazquez Roque M, Bouras EP. Epidemiology and management of chronic constipation in elderly patients. Clin Interv Aging. 2015;10:919-30. doi: https://doi.org/10.2147/ CIA.S54304.

13. Xu X, Pasricha PJ, Sallam HS, Ma L, Chen JD. Clinical significance of quantitative assessment of rectoanal inhibitory reflex (RAIR) in patients with constipation. J Clin Gastroenterol. 2008;42(6):692-8. doi: https://doi. org/10.1097/MCG.0b013e31814927ba.

14. Tetangco E, Yan Y, Rao SS. Performing and analyzing highresolution anorectal manometry. Neuro Gastro Latam Rev. 2018;2:123-32. doi: https://doi.org/10.24875/ NGL.19000016.

15. Carrington EV, Scott SM, Bharucha A, Mion F, RemesTroche JM, Malcolm A, et al. Expert consensus document: advances in the evaluation of anorectal function. Nat Rev Gastroenterol Hepatol. 2018;15(5):309-23. doi: https:// doi.org/10.1038/nrgastro.2018.27.

16. Lee HJ, Jung KW, Han S, Kim JW, Park SK, Yoon IJ, et al. Normal values for high-resolution anorectal manometry/topography in a healthy Korean population and the effects of gender and body mass index. Neurogastroenterol Motil. 2014;26(4):529-37. doi: https://doi.org/10.1111/ nmo. 12297.

17. Carrington EV, Brokjaer A, Craven H, Zarate N, Horrocks EJ, Palit S, et al. Traditional measures of normal anal sphincter function using high-resolution anorectal manometry (HRAM) in 115 healthy volunteers. Neurogastroenterol Motil. 2014;26(5):625-35. doi: https://doi.org/10.1111/ nmo. 12307.

18. Noelting J, Ratuapli SK, Bharucha AE, Harvey DM, Ravi K, Zinsmeister AR. Normal values for high-resolution anorectal manometry in healthy women: effects of age and significance of rectoanal gradient. Am J Gastroenterol. 2012;107(10):1530-6. doi: https://doi.org/10.1038/ ajg.2012.221.

19. Oblizajek NR, Gandhi S, Sharma M, Chakraborty S, Muthyala A, Prichard D, et al. Anorectal pressures measured with high-resolution manometry in healthy peopleNormal values and asymptomatic pelvic floor dysfunction. Neurogastroenterol Motil. 2019;31(7):e13597. doi: https://doi.org/10.1111/nmo.13597.

20. Kahrilas PJ, Bredenoord AJ, Fox M, Gyawali CP, Roman S, Smout AJ, et al. The Chicago Classification of esophageal motility disorders, v3.0. Neurogastroenterol Motil. 2015;27(2):160-74. doi: https://doi.org/10.1111/ nmo.12477. 